\title{
Linewidth and viscosity of isobutyric acid-deuterium oxide mixtures of critical composition above and below the phase separation temperature
}

\author{
B. Kalbskof and D. Woerman \\ Institut fur Physikalische Chemie, Universitat Koeln, Koeln, West Germany \\ B. Chu
}

Department of Chemistry, State University of New York at Stony Brook, Stony Brook, New York 11794

Erdogan Gularia)

Department of Chemical Engineering, The University of Michigan, Ann Arbor, Michigan 48109

(Received 5 December 1980; accepted 5 February 1981)

\begin{abstract}
Linewidth and visocity measurement covering the single phase and two phase region were made. Comparison of the data with the mode-mode coupling results of Kawasaki show good agreement with no adjustable parameters. In the one phase region measurements of autocorrelation functions indicate small deviations from a single exponential when $\left|T-T_{\mathrm{c}}\right| \leq 0.05^{\circ} \mathrm{C}$. The shear viscosity exponent $\phi$ was found to be $0.077 \pm 0.002$, somewhat larger than the theoretical estimates.
\end{abstract}

\section{INTRODUCTION}

In a previous paper ${ }^{1}$ results from static light scatter ing measurements and studies of the coexistence curve near the critical point of the system isobutyric acid/ $\mathrm{D}_{2} \mathrm{O}$ have been reported. This system is very interesting because of the presence of isotope exchange reactions. ${ }^{2}$ In this paper we present the results of dynamic light scattering measurements and shear viscosity studies in the neighborhood of the critical point for the same system.

Far away from the critical point of a binary mixture the half -width $\Gamma$ of the Rayleigh line is given by

$$
\Gamma=D k^{2},
$$

where $D$ is the mutual diffusion coefficient, $k=4 \pi \sin (\theta /$ 2) $/ \lambda$, with $\theta$ and $\lambda$ being the scattering angle and the wave length of light in the medium, respectively.

Equation (1) is valid only when the correlation length $\xi$ of the concentration fluctuations causing the scattering is much less than the reciprocal of the scattering vector $k$ (i.e., $k \xi \ll 1)$. Development ${ }^{3}$ and extension ${ }^{4}$ of the mode-mode coupling theory have made it possible to predict $\Gamma$ as function of $k \xi$ over the entire $k \xi(=X)$ range. Kawasaki first predicted

$$
\Gamma=\left(k_{B} T / 6 \pi \eta^{*} \xi^{3}\right) H_{0}(X),
$$

where $k_{B}$ is the Boltzmann constant, $T$ is the thermody namic temperature, $\eta^{*}$ is the high frequency shear viscosity, and the function $H_{0}(X)$ is given by

$$
H_{0}(X)=\frac{3}{4}\left[1+X^{2}+\left(X^{3}-X^{-1}\right) \arctan X\right] .
$$

In the critical region, $X \gg 1$, the linewidth is given by $\Gamma(K)=k_{B} T k^{3} / 16 \eta^{*}$. Far away from the critical point, $X \ll 1$, Eq. (2) reduces to Eq. (1). Kawasaki later introduced several improvements and obtained an expres sion for the high frequency viscosity ${ }^{5}$

\footnotetext{
a) Author to whom requests for reprints should be sent.
}

$$
\eta^{*}=\left[H_{0}(X) / H(X)\right] \eta \text {. }
$$

Numerical values of the ratio $H_{0}(X) / H(X)$ are given in Ref. 5. Further improvements, such as correlation function correction, vertex correction, etc., improve the already good agreement between theory and experi ment. 6

The shear viscosity of a binary liquid mixture near the critical mixing point exhibits an anomalous increase. Since the measured maximum increase $\Delta \eta / \eta$ is about $30 \%$ very near the critical point, it has not been possible to determine whether $\Delta \eta\left(=\eta-\eta_{0}\right.$ with $\eta_{0}$ being the nondevergent part of the shear viscosity) exhibits a power law divergence, a logarithmic divergence or just a finite cusp. Theoretically the predictions also range from a finite $\operatorname{cusp}^{7}$ to a weak power law. ${ }^{8}$

\section{MATERIALS AND METHODS}

\section{A. Materials}

Isobutyric acid (abbreviated $\mathrm{ICO}_{2} \mathrm{H}$ ) with a purity of $99 \%$ was fractionally distilled in a concentric tube column of 75 theoretical plates. Deuterium oxide $\left(\mathrm{D}_{2} \mathrm{O}\right)$, which was used without further purification, was of Merck Uvasol quality with a degree of deuterium of $99.75 \%$. The samples used for the light scattering experiments were the same as that used in our previous study. ${ }^{2}$ All the samples had compositions very close to the critical composition; mass fraction of the acid $\left(y_{c}\right)=0.356$.

\section{B. Light scattering}

The experimental arrangement of our digital light scattering photometer has been described elsewhere. ${ }^{\circ}$ In order to insure long term thermal stability and uni formity, three cascaded temperature controllers were used for temperature control. In addition, the final controller was housed in a thermostated box in order to eliminate drifts due to room temperature variations. Temperature of the sample was controlled to $0.001^{\circ} \mathrm{C}$ 
and measured with a resolution of $0.0001^{\circ} \mathrm{C}$.

Linewidth measurements were made as a function of time and height. In the one phase region, a minimum of $3 \mathrm{~h}$ was allowed for thermal equilibrium. In the two phase region, the final data were taken after it was established that the linewidth of the autocorrelation function was not changing with respect to time. This meant an average of $24-36 \mathrm{~h}$ of waiting after changing the sample temperature. Close to the phase separation temperatures waiting times were much longer. As another check of equilibrium, linewidth of the autocorrelation function was also measured as a function of height difference from the meniscus.

\section{Viscosity}

The Ubbelohde type viscometer used in this study is shown schematically in Fig. 1. It allows measurements of the kinematic viscosity of the homogeneous mixture as well as that of the coexisting fluid phases in a closed system. The viscometer, filled with about $20 \mathrm{~cm}^{3}$ of a mixture of critical composition, was flame sealed. The change in composition of the mixture in the viscometer due to evaporation into its dead space could be neglected. It was mounted on a rack which allowed the viscometer to be turned by an angle of $360^{\circ}$ and positioned again reproducibly in the vertical position. The rack with the viscometer was immersed into a carefully controlled thermostat (long time temperature stability $\delta T=2.10^{-3}$ $\mathrm{K}$ in a temperature range $25^{\circ}-60^{\circ} \mathrm{C}$ ). By rotating the viscometer, it was possible to move the mixture from the lower part of the viscometer to its upper part before starting a measurement. The crucial part of the viscometer is the stopcock which was used for separating the two coexisting fluid phases at temperatures below the phase separation temperature. The stopcock (Corning, Rotaflo) consists of a hollow, thin walled Teflon stopper which fits into a precision-bore glass tube of slightly bigger diameter. The stopper contains a rubber plug which can be pressed together between two metal O-rings by turning a screw. In this way the diameter of the plug is increased and the wall of the stopper is pressed against the interior of the glass tube making a tight seal. The glass tube is provided with a thread at its upper end. The head of the stopper functions as a nut for the glass thread moving the stopper back and forth. To make sure that no thermostating fluid could penetrate into the interior of the viscometer the Teflon stopper had an additional tightly fitting O-ring in its upper part. In this way another seal between the stopper and the glass tube was made. The fact that temperature of phase separation of the sample within the viscometer did practically not change during the experiments (at the beginning $T_{p}=44.947^{\circ} \mathrm{C}$; at the end $T_{p}=44.943^{\circ} \mathrm{C}$ ) indicates tightness of the seals.

The viscosity of the mixtures was calculated from the experimentally determined efflux time of the fluid contained in the volume above the capillary using Eq. (5):

$$
\eta / \rho=a t-b / t^{2},
$$

where $\eta$ is the viscosity, $\rho$ is the density, $t$ is the efflux time, and $a$ and $b$ are constants. The viscometer was

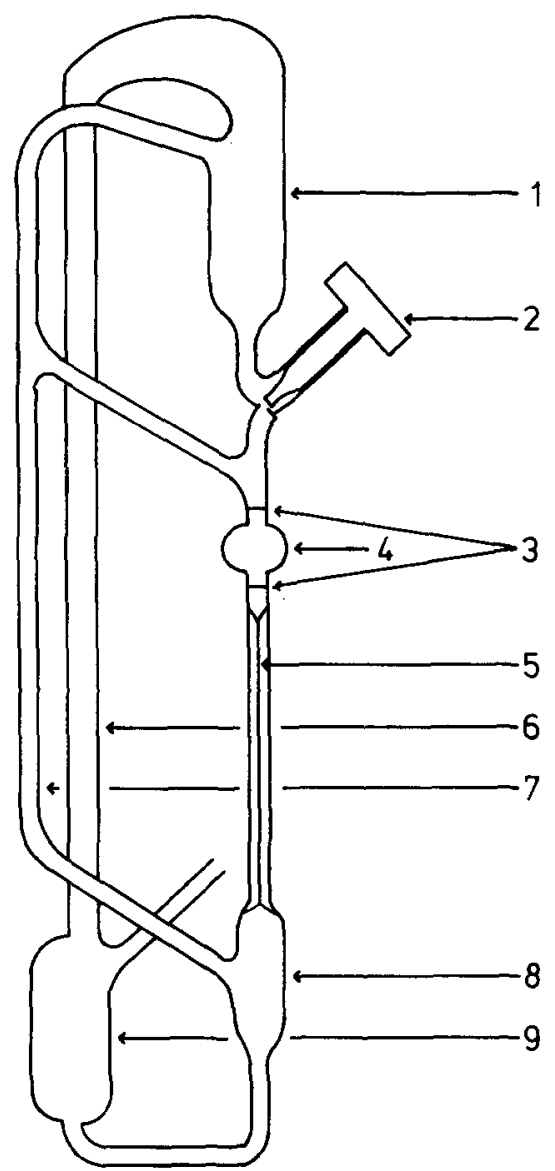

FIG. 1. Modified Ubbelhode capillary viscometer used for measuring viscosities. 1 -volume $\sim 20 \mathrm{~cm}^{3}, 2$-stopcock, 3timing marks, 4-timed volume $\sim 3.5 \mathrm{~cm}^{3}$, 5-capillary tube $0.43 \mathrm{~mm}$ diam, 6-tubing, diam $1 \mathrm{~cm}, 7$-tubing diam $0.5 \mathrm{~cm}$, 8 -volume $\approx 20 \mathrm{~cm}^{3}$.

calibrated with water. The efflux time of water at $30^{\circ} \mathrm{C}$ was about $250 \mathrm{~s}$ and at $60^{\circ} \mathrm{C}$ about $140 \mathrm{~s}$. The constants $a$ and $b$ had the values $a=3.528 \times 10^{-5} \mathrm{~cm}^{2} \mathrm{~s}^{-2}$ and $b=1.200$ $\times 10^{-2} \mathrm{~cm}^{2}$. It was found that $a$ and $b$ did not change with temperature in the temperature range given.

\section{Density}

The densities of the mixture above the phase separation temperature and the densities of the coexisting fluid phases below the phase separation temperature were measured, respectively, in a digital precision densimeter (Paar, Typ DMA 02). They were calculated from the electronically measured frequency of a mechanical oscillator filled with the mixture. The oscillator is a $\mathrm{U}$-shaped glass tube (volume about $0.7 \mathrm{~cm}^{3}$ ) placed in a metal block whose temperature was controlled. The instrument was calibrated in the temperature range $30^{\circ}-50^{\circ} \mathrm{C}$ using water and air as reference substances. Special care was taken to seal the oscillator tube air tight in order to prevent evaporation of its contents. The densities of the coexisting fluid phases below the phase separation temperature were measured in the fol lowing way. Samples of critical composition were prepared by weighing in the appropriate amounts of the two components into suitable tubes and sealing them with 
silicone rubber caps to make them airtight. These samples were kept at a different temperature $T^{*}$ below the phase separation temperature $T_{P}$. After establishment of the phase equilibrium between the coexisting fluid phases samples of the two phases were taken using Hamilton syringes whose glass bodies and pistons were kept at a temperature above the temperature $T^{*}$. The density of each sample was measured as a function of temperature in the temperature range $\Delta T=T-T^{*}$ up to about $1^{\circ} \mathrm{C}$ above $T^{*}$. The density at $T^{*}$ was obtained by extrapolation so $\Delta T=0$.

\section{DISCUSSION AND RESULTS}

\section{A. Light scattering results: single phase $\left(T>T_{c}\right)$}

All of the one phase linewidth measurements obtained in five different experimental runs are shown in Fig. 2. Also shown in Fig. 2 is a plot of Eq. (2) including the nonlocal viscosity correction given by Eq. (4) using experimentally determined values of the correlation length $\xi$ and the viscosity $\eta$. We see that over the temperature range covered $\left(20 \geq \Delta T \geq 0.004^{\circ} \mathrm{C}\right)$, the agreement be tween theory and experiment is fairly good. We should point out that there are no adjustable parameters either in the theoretical curve or the experimental data. There is some scatter around $K \xi \sim 1$ as has been observed before. ${ }^{6}$

One of our aims was to try to detect whether the theoretically predicted deviation of the autocorrelation function from a single exponential could be observed. Deviations from a single exponential curve could be measured quantitatively by fitting the autocorrelation function to a modified nonlinear cumulants expansion in order to obtain the variance of the distribution of exponentials about the mean. ${ }^{10}$ The results are shown in Fig. 4. For $\Delta T$ values greater than $0.5^{\circ} \mathrm{C}$, the vari-

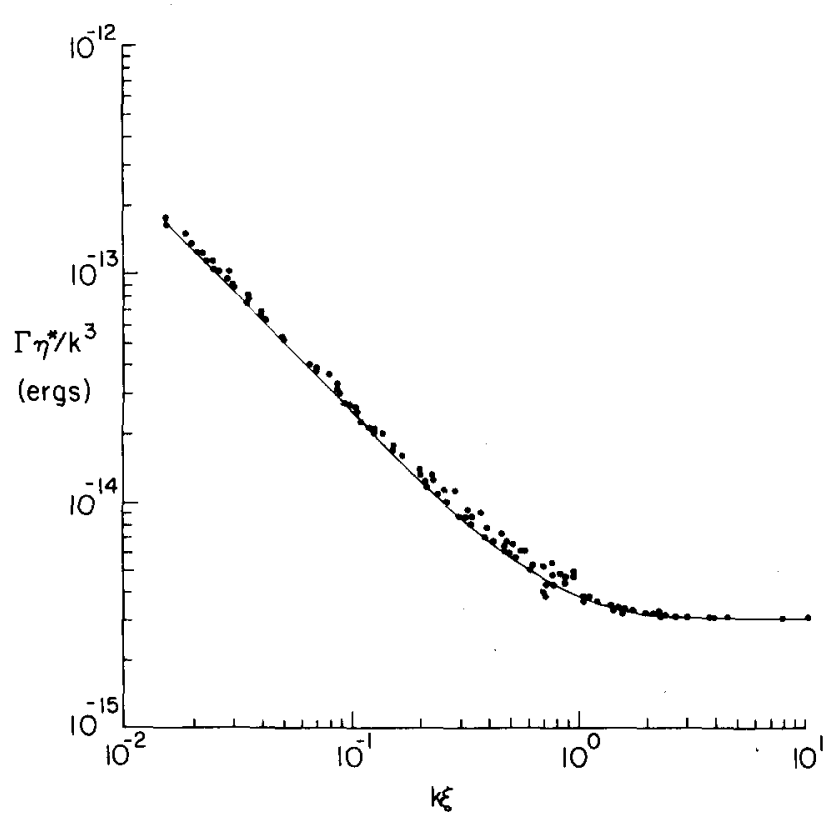

FIG. 2. Plot of linewidth data in the one phase region. The solid line represents Eqs. (2) and (4) using experimentally determined values of $\xi$ and $\eta$.
TABLE I. Measurement of linewidth in the one-phase region as a function of height. Total sample height $=20 \mathrm{~mm}$. Height of the visible section $12.5 \mathrm{~mm}$. Heights are given from the bottom of the observation section.

\begin{tabular}{lllll}
\hline$\theta(\mathrm{deg})$ & $\Delta T\left({ }^{\circ} \mathrm{C}\right)$ & $\Gamma(5 \mathrm{~mm})$ & $\Gamma(7.5 \mathrm{~mm})$ & $\Gamma(10 \mathrm{~mm})$ \\
\hline 36.4 & 4.5 & $2515 \pm 20$ & $2545 \pm 20$ & $2543 \pm 20$ \\
36.4 & 1.85 & $787 \pm 8$ & $793 \pm 8$ & $799 \pm 10$ \\
36.4 & 0.020 & 123 & 123 & $\cdots$ \\
\hline
\end{tabular}

ance reaches a limiting value of $2 \%$ which is equivalent to the error introduced due to statistical uncertainties. As $\Delta T$ decreases, the variance increases by about fivefold to $10 \%$ at $\Delta T=0.004^{\circ} \mathrm{C}$. For this scattering angle $\left(\theta=144^{\circ}\right)$, the linewidth $\Gamma$ changes by less than $30 \%$ over the $\Delta T$ range shown. Therefore we feel that the observed increase in the variance of the autocorrelation functions is real and cannot be due to short term fluctuations $\left( \pm 0.0005^{\circ} \mathrm{C}\right.$ or less) in the sample temperature. There is a small possibility that multiple scattering could have caused the deviation from a single exponential behavior. The maximum attenuation in our system was $\simeq 10 \%$ for $\Delta T \simeq 0.004^{\circ} \mathrm{C}$. As a result the multiple scattering effects are expected to be negligible. ${ }^{11}$

In order to minimize the effects of heating by the laser beam, we used a $15 \mathrm{~mW} \mathrm{He-Ne} \mathrm{laser} \mathrm{and} \mathrm{regulated} \mathrm{the}$ intensity reaching the sample by a polarizer right before the sample. The power going through the sample was never more than $\sim 7 \mathrm{~mW}$ and close to the critical point the power was lowered to about $\sim 0.5 \mathrm{~mW}$. The magnitude of heating by the laser beam was checked at several temperatures by measuring the linewidth. We obtained the following representative results: at $\Delta T$ $=0.338^{\circ} \mathrm{C}, \Gamma\left(\theta=36.4, I_{0}=1.5 \mathrm{~mW}\right)=434 \pm 3$ and $\Gamma(0.7$ $\mathrm{mW})=430 \pm 4$ with $d \Gamma / d(\Delta T) \sim 700$. This change in $\Gamma$ corresponds to $\sim 0.7 \mathrm{mK} / \mathrm{mW}$. At $\Delta T=0.034, \Gamma(\theta$ $\left.=36.4, I_{0}=7 \mathrm{~mW}\right)=143$ and $\Gamma(0.7 \mathrm{~mW})=134$ with $d \Gamma /$ $d(\Delta T) \sim 1200$; this again yields a heating due to the laser beam of $\sim 0.6 \mathrm{mK} / \mathrm{mW}$, which is comparable to other reported values. ${ }^{12}$ We made routine measurements of the autocorrelation function as a function of height in the sample cell in order to check whether there were vertical temperature and/or concentration gradients. The results, as shown in Table $I$, indicate that vertical gradients within the scattering cell are negligible.

\section{B. Two phase region}

All of our linewidth measurements in the two phase region are shown in Fig. 3 along with the theoretical predictions of Eqs. (2) and (4). Again neither the data nor the theoretical function have any adjustable parameters. Due to the fact that it is more difficult to get a reliable data point in the two phase region, the amount of data is less and there is some scatter. The taking of data at $\Delta T$ values less than $0.05^{\circ} \mathrm{C}$ was practically impossible due to long equilibration times of three to four days during which time even a temperature drift of $1 \mathrm{mK}$ or short term fluctuations created concentration gradients around the interface. Intensity in the one phase region was high enough to measure a correlation function at $\Delta T \sim 20^{\circ} \mathrm{C}$. In comparison, the largest $\Delta T$ 


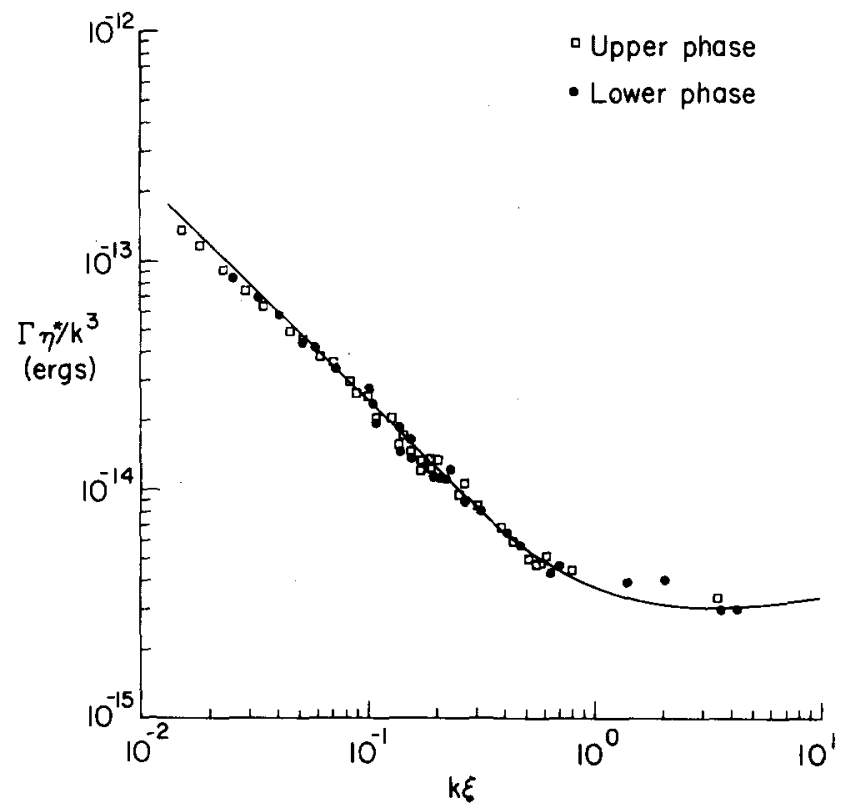

FIG. 3. Plot of linewidth data in the two liquid phase region: o upper phase, - lower phase. The solid line represents Eqs. (2) and (4) using the experimentally determined values of $\xi$ and $\eta$.

value at which we were able to make a linewidth measurement is $4^{\circ} \mathrm{C}$ in the two phase region. This is not surprising because each phase is moving from the critical composition and the critical temperature simultaneously. Finally we want to point out that for the same $\Delta T$, the lower phase $\left(\mathrm{D}_{2} \mathrm{O}\right.$ rich) has the larger linewidth but due to the much higher viscosity of the upper phase, the product $\Gamma \eta^{*}$ is larger for the upper phase.

\section{Viscosity results}

The shear viscosity of isobutyric acid/ $\mathrm{D}_{2} \mathrm{O}$ mixtures of critical composition shows a weak anomaly approaching the critical temperature from the one fluid phase region (as shown in Fig. 5). This behavior is similar

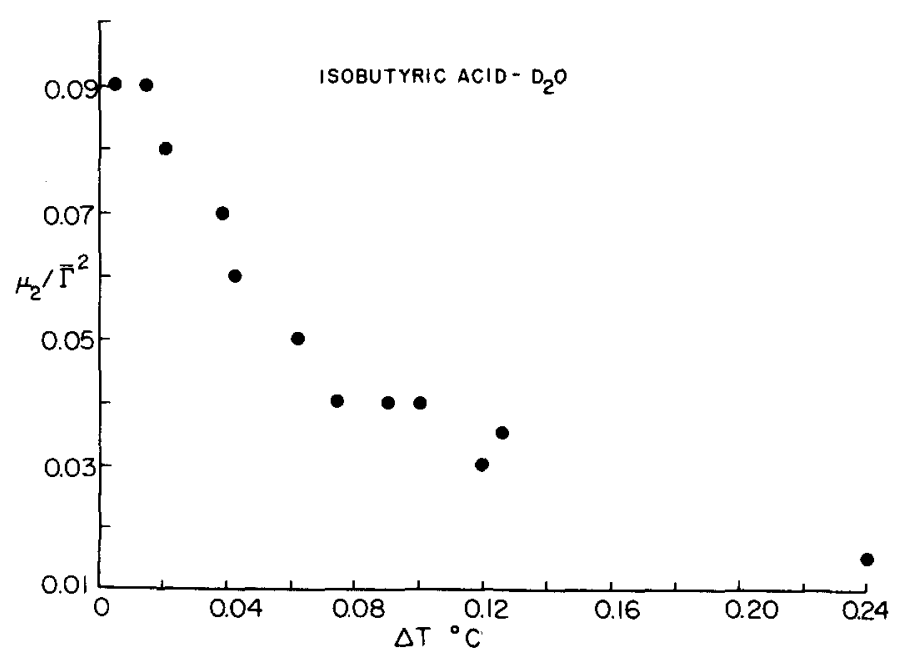

FIG. 4. Plot of variance of the autocorrelation function as a function of $\Delta T=\left(T-T_{c}\right)$.

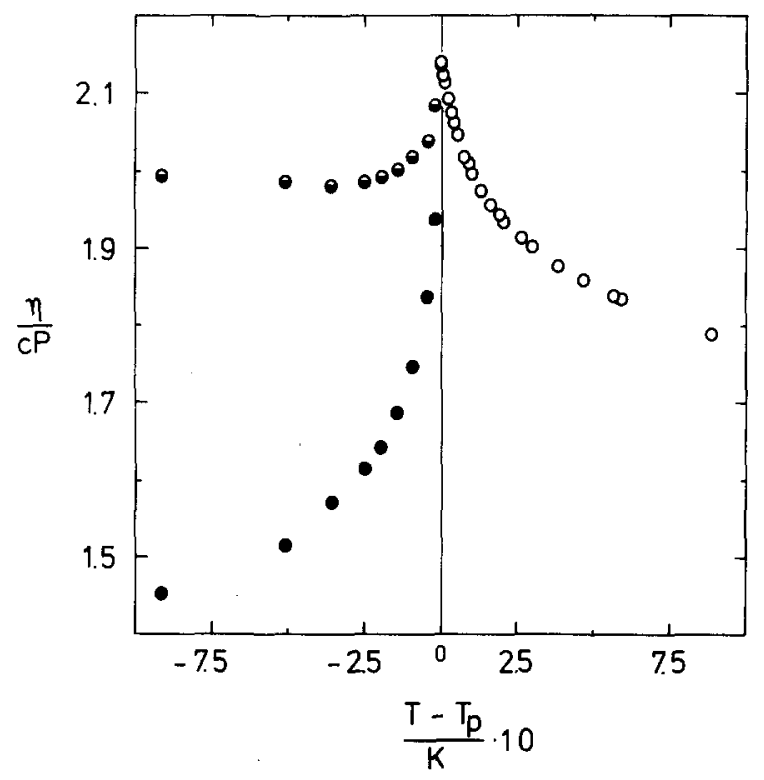

FIG. 5. Plot of viscosity data as a function of temperature. o Single phase region; o upper phase and -lower phase.

to that of one component systems ${ }^{19}$ and other binary and ternary critical mixtures. ${ }^{19,14}$ The shear viscosity of the coexisting fluid phases below $T_{c}$ also shows a weak anomaly approaching $T_{c}$ (see Fig. 5). Only the viscosity data obtained at temperatures above $T_{c}$ are analyzed in terms of the theory of dynamic critical phenomena. ${ }^{7,8,15}$ An analysis of the viscosity data of the coexisting fluid phases is not possible at present because the composition of the coexisting phases is not known. The system isobutyric acid $/ \mathrm{D}_{2} \mathrm{O}$ is a binary mixture of a special kind. Isobutyric acid is a weak acid so that isotope exchange reactions occur between the proton of the acid and the

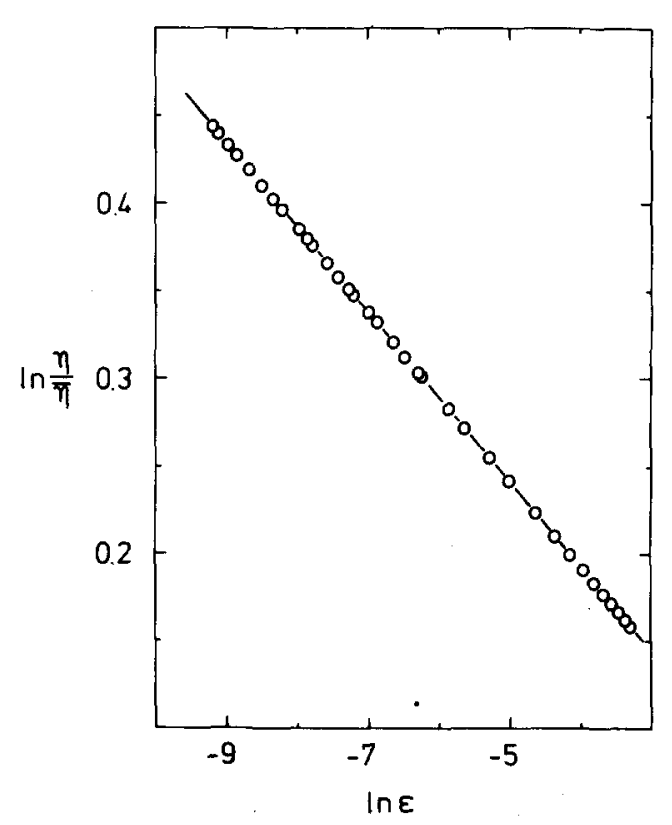

FTG. 6. Double logarithm plot of viscosity versus reduced temperature difference $\epsilon=\left[\left(T-T_{c}\right) / T_{c}\right]$. The solid line is given by $\eta=\epsilon^{-0.048} \exp (-6.32 \epsilon+0.32)$. 


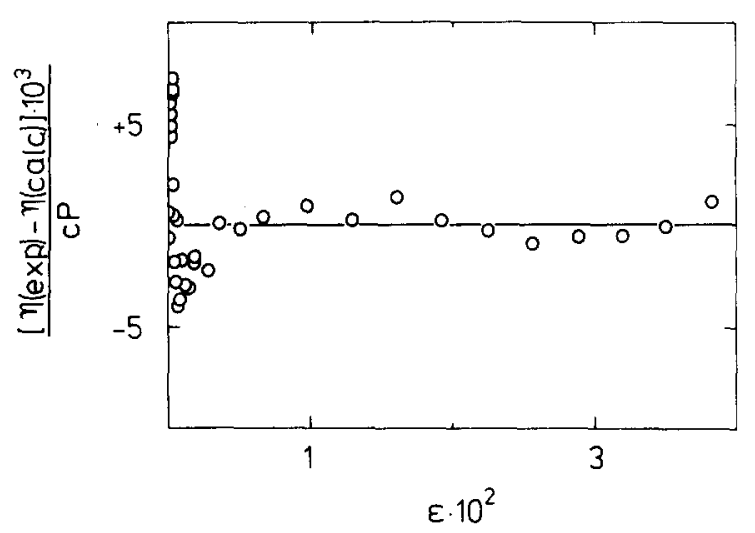

FIG. 7. Residuals of the curve fit shown in Fig. 6.

deuterium of the deuterium oxide. Consequently more than two species are present in the system in appreci able amounts $\left(\mathrm{D}_{2} \mathrm{O}, \mathrm{HDO}, \mathrm{DA}\right)$. Nevertheless, the system remains a two component system thermodynamically at temperatures $T>T_{c} \cdot{ }^{2}$ But at temperatures $T<T_{c}$, each of the coexisting phases is a three component system.

An empirical function [see Eq. (6)] proposed by Debye et $a l .{ }^{16}$ is fitted to the experimental viscosity data obtained at temperatures above $T_{c}$ using a weighted nonlinear least square fit program. The data were not corrected for nonlinear shear gradient effects.

$$
\eta / \eta^{+}=\epsilon^{-\Phi} \exp (B \epsilon+C) \text {. }
$$

$\eta$ is the shear viscosity measured in units of $\eta^{+}=1 \mathrm{cP}$ and $\epsilon$ is the reduced temperature $\epsilon=\left(T-T_{c}\right) / T_{c} . \phi$ is a critical exponent. $B$ and $C$ are constants independent of temperature. Equation (6) implies a power law of the form $\eta / \bar{\eta}=\epsilon^{-\Phi} . \bar{\eta}$ is the shear viscosity in absence of any critical anomaly. Furthermore, it is assumed that

TABLE II. Viscosity $\eta$ of an isobutyric acid/ $\mathrm{D}_{2} \mathrm{O}$ mixture of near critical composition $\left(y=0.355_{2}\right.$; $y=$ mass fraction of isobutyric acid) at different temperature differences $\left(T-T_{p}\right)$ above the phase separation temperature $T_{p}\left(T_{p}=44.945^{\circ} \mathrm{C}\right)$.

\begin{tabular}{llll}
\hline \hline$\left(T-T_{p}\right) / K$ & $\eta / \mathrm{cP}$ & $\left(T-T_{0}\right) / K$ & $\eta / \mathrm{cP}$ \\
\hline 12.176 & 1.2623 & 0.300 & 1.9016 \\
11.140 & 1.2930 & 0.262 & 1.9123 \\
10.153 & 1.3242 & 0.206 & 1.9338 \\
9.169 & 1.3571 & 0.192 & 1.9443 \\
8.137 & 1.3931 & 0.161 & 1.9565 \\
7.138 & 1.4308 & 0.133 & 1.9735 \\
6.119 & 1.4716 & 0.102 & 1.9970 \\
5.101 & 1.5162 & 0.092 & 2.0111 \\
4.102 & 1.5619 & 0.079 & 2.0177 \\
3.105 & 1.6154 & 0.056 & 2.0459 \\
2.116 & 1.6775 & 0.045 & 2.0603 \\
1.596 & 1.7173 & 0.034 & 2.0743 \\
1.119 & 1.7633 & 0.023 & 2.0925 \\
0.888 & 1.7884 & 0.014 & 2.1121 \\
0.596 & 1.8331 & 0.009 & 2.1224 \\
0.569 & 1.8377 & 0.004 & 2.1322 \\
0.467 & 1.8569 & 0.001 & 2.1401 \\
0.385 & 1.8763 & & \\
\hline \hline
\end{tabular}

TABLE III. Viscosities $\eta$ of coexisting phases of an isobutyric acid $/ \mathrm{D}_{2} \mathrm{O}$ mixture of near critical composition $(y$ $=0.355_{2} ; y=$ mass fraction of isobutyric acid) at different temperature differences $\left(T_{p}=T\right)$ below the phase separation temperature $T_{p}\left(T_{p}=44.945^{\circ} \mathrm{C}\right) u$-phase = upper phase (high isobutyric acid con tent), $l$-phase $=$ lower phase (low isobutyric acid content), $1 \mathrm{cP}=10^{5} \mathrm{~J} \mathrm{~s} \mathrm{~cm}^{-1}$.

\begin{tabular}{lll}
\hline \hline$\left(T_{p}-T\right) / K$ & \multicolumn{2}{c}{$\eta / \mathrm{cP}$} \\
\hline & $u$-phase & $l$-phase \\
0.018 & 2.1044 & 1.9367 \\
0.041 & 2.0586 & 1.8366 \\
0.090 & 2.0182 & 1.7454 \\
0.142 & 2.0012 & 1.6850 \\
0.192 & 1.9920 & 1.6412 \\
0.248 & 1.9863 & 1.6148 \\
0.357 & 1.9812 & 1.5710 \\
0.505 & 1.9851 & 1.5155 \\
0.910 & 1.9946 & 1.4521 \\
1.433 & 2.0072 & 1.4057 \\
1.896 & 2.0352 & 1.3752 \\
2.892 & 2.0837 & 1.3412 \\
3.886 & 2.1316 & 1.3267 \\
4.898 & 2.1879 & 1.3207 \\
5.897 & 2.2333 & 1.3224 \\
6.858 & 2.2931 & 1.3295 \\
7.903 & 2.3414 & 1.3403 \\
8.900 & 2.4050 & 1.3547 \\
9.852 & 2.4470 & 1.3701 \\
10.889 & 2.5079 & 1.3899 \\
11.838 & 2.5612 & 1.4110 \\
12.881 & 2.6361 & 1.4348 \\
14.917 & 2.7503 & 1.4910 \\
\hline \hline
\end{tabular}

TABLE IV. Density $\rho$ of an isobutyric acid/ $\mathrm{D}_{2} \mathrm{O}$ mixture of near critical composition ( $v$ $=0.355_{2} ; y=$ mass fraction of isobutyric acid) at different temperature differences $\left(T-T_{p}\right)$ above the phase separation temperature $T_{p}\left(T_{p}=45.05^{\circ} \mathrm{C}\right)$.

\begin{tabular}{ll}
\hline \hline$\left(T-T_{p}\right) / K$ & $\rho / \mathrm{g} \mathrm{cm}^{-3}$ \\
\hline 5.022 & 1.403770 \\
4.488 & 1.044122 \\
3.993 & 1.044532 \\
3.489 & 1.044896 \\
2.989 & 1.045255 \\
2.479 & 1.045617 \\
2.087 & 1.045890 \\
1.682 & 1.046171 \\
1.287 & 1.046451 \\
0.873 & 1.046736 \\
0.674 & 1.046865 \\
0.483 & 1.046994 \\
0.312 & 1.047106 \\
0.319 & 1.047105 \\
0.178 & 1.047198 \\
0.069 & 1.047266 \\
\hline \hline
\end{tabular}


TABLE V. Densities $\rho$ of coexisting phases of an isobutyric acid $/ \mathrm{D}_{2} \mathrm{O}$ mixture of near critical composition at difrerent temperature differences $\left(T_{p}-T\right)$ below the phase separation temperature $T_{p} ; y=$ mass fraction of isobutyric acid. $u$-phase $=$ upper phase (high isobutyric acid content), $l$-phase $=$ lower phase (low isobutyric acid content).

\begin{tabular}{llll}
\hline \hline$\left(T_{p}-T\right) / K$ & $y$ & \multicolumn{2}{c}{$\rho / \mathrm{g} \mathrm{cm}^{-3}$} \\
\hline 0.120 & $0.355_{2}$ & 1.03359 & 1.50874 \\
1.137 & $0.355_{1}$ & 1.02739 & 1.06493 \\
2.182 & $0.355_{1}$ & 1.02412 & 1.06891 \\
3.174 & $0.356_{1}$ & 1.02206 & 1.07134 \\
4.185 & $0.355_{3}$ & 1.02045 & 1.07334 \\
5.250 & $0.355_{3}$ & 1.01875 & 1.07507 \\
6.257 & $0.355_{3}$ & 1.01737 & 1.07646 \\
7.277 & $0.355_{1}$ & 1.01647 & 1.07812 \\
8.349 & $0.355_{0}$ & 1.01578 & 1.07939 \\
9.362 & $0.355_{3}$ & 1.01461 & 1.08068 \\
10.425 & $0.355_{3}$ & 1.01376 & 1.08173 \\
11.592 & $0.355_{3}$ & 1.01307 & 1.08284 \\
12.632 & $0.355_{2}$ & 1.01251 & 1.08382 \\
13.780 & $0.355_{2}$ & 1.01191 & 1.08470 \\
15.046 & $0.355_{2}$ & 1.01136 & 1.08571 \\
\hline \hline
\end{tabular}

the temperature dependence of $\bar{\eta}$ can be described by an Arrhenius type equation $\left[\bar{\eta}=\exp \left(B_{e}+C\right)\right]$.

The best fit of Eq. (6) to the experimental data $\left(\chi_{r}^{2}\right.$ $=1.71$ ) was obtained with a critical temperature $T_{c}$ $=44.913^{\circ} \mathrm{C}$. This value is $32 \mathrm{mK}$ lower than the visual ly determined value of $T_{p}=44.945^{\circ} \mathrm{C}$. The parameters $B, C$, and $\phi$ have the values $C=0.32 \pm 0.01, B=-6.32$ $\pm 0.03, \phi=0.048 \pm 0.001$. Figure 6 shows a plot of $\ln \eta / \bar{\eta}$ vs $\ln \epsilon$. According to Eq. (6), a linear relationship is expected. The critical exponent $\alpha$ is given by the slope of the straight line. The residuals of the fit of Eq. (6) to the experimental data are shown in Fig. 7. The $\phi$ value found for the system isobutyric acid/ $\mathrm{D}_{2} \mathrm{O}$ is close to the best experimental estimate of $\phi=0.0398$ \pm 0.0005 . $^{16-18}$

More recent theoretical studies of the shear viscosity near the critical point suggest a relation of the form

$$
\eta / \hat{\eta}=(q \xi)^{\circ}
$$

to describe the temperature dependence of the shear vis cosity. ${ }^{7,19,20}$ In Eq. (7), $\xi=\xi_{0} \epsilon^{-\nu}$, where $\xi_{0}$ is the critical amplitude and $\nu$ the critical exponent of the correlation length $(\nu \simeq 0.62) . q$ is an undetermined system dependent constant. $\hat{\eta}$ is a background shear viscosity which remains finite at the critical point. Several alternative attempts have been made to calculate the ex ponent $\phi^{\prime}$ from theory. The theoretical values currently available lay in the range $0.054<\phi^{\prime}<0.065$. Equation (7) is equivalent to the empirical Eq. (6) with

$$
\phi=\phi^{\prime} \nu, \quad \hat{\eta}_{\phi^{\prime}}=\exp \left(B \epsilon+C^{\prime}\right), \quad\left(\xi_{0} q\right)=\exp \left(C-C^{\prime}\right) \text {. }
$$

With $\nu=0.62, \phi^{\prime}=0.077 \pm 0.002$ for the system isobutyric acid $/ \mathrm{D}_{2} \mathrm{O}$. This value is about $20 \%$ larger than the highest theoretical estimates of $\phi^{\prime}$. No explanation can be given for this finding. However, a similar high value of $\phi^{\prime}$ has recently been reported for the system nitrobenzene $/ n$-heptane with $\phi^{\prime}=0.073(\phi=0.045$ $\pm 0.013) .^{21}$ We also used a more general equation to analyze the viscosity data

$$
\Delta \eta=\eta-\bar{\eta}=(H / \phi)\left(\epsilon^{\phi}-1\right)+G .
$$

Equation (8) corresponds to a cusp for $0<\phi<1$, a power law divergence for $\phi<0$ and a logarithmic divergence for $\phi=0 .{ }^{22}$ A nonlinear least squares fitting of $\mathrm{Eq} .(8)$ to the one phase data yielded $\phi=0.22 \pm 0.05$ indicating a finite cusp behavior. The experimental values of vis cosity and density are given in Tables $\Pi-V$.

\section{ACKNOWLEDGMENTS}

We thank M. Pelger for his help in constructing the viscometer and in evaluating the experimental data. Partial support of this research was given by the National Science Foundation and is gratefully acknowledged.

${ }^{1}$ E. Gulari, B. Chu, and D. Woerman, J. Chem. Phys. 73, $2480(1980)$

${ }^{2}$ P. Gauser, T. Jansen, W. Schon, D. Woerman, and H. Schoenes, Ber. Gunsenges. Phys. Chem. (to be published).

${ }^{3}$ L. P. Kadanoff and J. Swift, Phys. Rev. 116, 89 (1968).

${ }^{4}$ K. Kawasaki, Phys. Lett. 30A, 325 (1969).

${ }^{5}$ K. Kawasaki and S. M. Lo, Phys. Rev. Lett. 29, 48 (1972).

${ }^{6}$ B. Chu, S. P. Lee, and W. Tscharnuter, Phys. Rev. A 7, 353 (1973).

${ }^{7}$ K. Kawasaki, Ann. Phys. 61, 1 (1970).

${ }^{8}$ B. I. Halperin, P. C. Hohenberg, and E. D. Siggia, Phys. Rev. Lett. 32, 1289 (1974); E. D. Siggia, B. I. Halperin, and P. C. Hohenberg, Phys. Rev. B 13, $2110(1976)$.

${ }^{9}$ B. Chu, E. Gulari, and E. Gulari, Phys. Scr. 19, 476 (1979).

${ }^{10}$ D. E. Koppel, J. Chem, Phys. 57, 4814 (1972).

${ }^{11}$ D. S. Siano, B. J. Berne, and G. W. Flynn, J. Coll. Interface Sci. 63, 282-289 (1978).

${ }^{12}$ R. F. Chang, H. Burstyn, and J. V. Sengers, Phys. Rev. A 19, 866 (1979); C. M. Sorensen, R. C. Mockler, and W. J. O'Sullivan, Phys. Rev. Lett. 40, 777 (1978).

${ }^{13}$ J. V. Sengers, Ber. Bunsenges. Phys. Chem. 65, 234 (1972).

${ }^{14}$ S. P. Lee and A. J. Purvis, Chem. Phys. 24, 191 (1977).

${ }^{15}$ P. C. Hohenberg and B. I. Halperin, Rev. Mod. Phys. 49, 435 (1977).

${ }^{16}$ P. Debye, B. Chu, and D. Woermann, J. Polym. Sci. Part A 1, 249 (1963).

${ }^{17}$ P. Calmettes, Phys. Rev. Lett. 39, 1151 (1977); J. Phys. Lett. 40, 535 (1979).

${ }^{18}$ S. P. Lee, Chem. Phys. Lett. 25, 611 (1978).

${ }^{19}$ R. Perl and R. A. Ferrel, Phys. Rev. Lett. 40, 777 (1978).

${ }^{20}$ D. W. Oxtoby and W. M. Gelbart, J. Chem. Phys. 61, 2957 (1974).

${ }^{21}$ G. Z. Zalczer and D. Beysens, J. Chem. Phys. 72, 348 (1980).

${ }^{22} \mathrm{~J}$. V. Sengers, University of Maryland Preprints, Tech. Report No. 71-074. 